\title{
RAPID PRODUCTION OF GRAPHITE WITHOUT CONTAMINATION FOR BIOMEDICAL AMS
}

\section{JOHN S. VOGEL}

Center for Accelerator Mass Spectrometry, University of California, Lawrence Livermore National Laboratory, Box 808 L-397, Livermore, California 94551 USA

\begin{abstract}
The application of AMS to the detection of ${ }^{14} \mathrm{C}$ makes possible a new class of sensitive experiments in molecular biology. Such experiments inherently produce large numbers of samples for the determination of biological variability in molecular interactions. The samples vary in ${ }^{14} \mathrm{C}$ concentration over many orders of magnitude. We added $\mathrm{TiH}_{2}$ to aid the reduction of $\mathrm{CO}_{2}$ by zinc in a sealed tube to reproducibly make graphite without sample cross-contamination. to aid the reduction of $\mathrm{CO}_{2}$ is transferred from a combustion tube to the reaction tube through a disposable plastic manifold. The sealed tubes are heated to a single-reaction temperature in a muffle furnace. The process is complete within $5 \mathrm{~h}$. Bulk isotopic fractionation in the finished graphite is less than $0.5 \%$.
\end{abstract}

\section{INTRODUCTION}

A LLNL Biomedical Sciences group began AMS ${ }^{14} \mathrm{C}$ measurements of biomedical research materials in 1988. Our initial experiments are directed toward detecting covalent binding of ${ }^{14} \mathrm{C}$-labeled molecules to DNA as an indicator of potential genotoxic effects that produce mutations or cancers (Turteltaub et al. 1990), understanding pharmacokinetics of toxins and chemotherapeutics and developing a sensitive radioimmunoassay for environmental or clinical surveys. The primary technical task in making the sensitivity of AMS available for such biomedical research was the preparation of samples in a form suitable for ionization in an AMS ion source. The large numbers and different types of samples from our current research provide the initial conditions that a preparation process must meet.

We trace xenobiotics (chemicals not native to the body, whether toxic or beneficial) by introducing a labeled compound into a biological system (primarily mice or cell cultures), followed by dissecting or isolating one or more parts of the system to determine the concentration of the compound present in those parts. A series of such tests provides a time history of the spread of the compound, if the time between the initial dosing and sample isolation is varied. Applying AMS to tracer studies improves sensitivity by five or six orders of magnitude, making possible the testing of many toxins at environmentally relevant levels. Since AMS uses only $1 \mathrm{mg}$ or less of extracted carbon, repeated measurements can be made on the same tissue. The ${ }^{14} \mathrm{C}$ label is in a stable location within the molecule, so that stoichiometric identity is maintained between the ${ }^{14} \mathrm{C}$ measured above contemporary levels and the carcinogen concentration. For example, we traced ${ }^{14} \mathrm{C}$-labeled phenyl-imidazo-pyridine through the blood, excreta and 11 organs or tissues in order to obtain the murine response to a single dose of this potent carcinogen found in cooked muscle meats. Three mice were analyzed at each of 16 time delays after dosing. The experiment produces at least 624 separate samples, but the number exceeds 800 when duplicates for testing or redilutions for more precision are included. The ${ }^{14} \mathrm{C}$ concentrations range from contemporary to several hundred times Modern; the "hottest" samples are the urine shortly after dosing. Many of the tissues rise above contemporary ${ }^{14} \mathrm{C}$ levels by factors of only $0.3-2$, but the signal indicating their exposure to the carcinogen is distinct and quantifiable.

Such complete tracing of a carcinogen has never before been accomplished, since no initial dose of ${ }^{14} \mathrm{C}$-labeled carcinogen could have been detected in all the tissues of interest by decay counting without incurring tissue damage through the sheer amount of the administered compound. In 
previous tests (Turteltaub et al. 1989), a detectable dose of carcinogen would be equivalent to that found in 80 million hamburgers, whereas this experiment uses the equivalent of only a single hamburger's toxin. We measured samples from this study at the rate of 20-40 per week, and required several months to complete the AMS measurements.

The development of a sensitive, competitive radioimmunoassay is another example of sample preparation and measurement designed for AMS-related biological research. This development will provide a tool for quantifying trace compounds in humans and in environments at low levels without directly using radio-labeled compounds. We produce 20-30 samples per week to discover the binding characteristics of the antibodies at low concentrations of labeled and unlabeled reactants. The measurements must be made shortly after the assay experiments, so that further design of the assay can be based upon these results. For this reason, we use the AMS facility every Monday afternoon for biomedical measurements. We analyze and discuss the data on Tuesdays. We perform new assays on Wednesdays. The samples are diluted, dried, combusted and graphitized on Thursdays. We mount them in the sample holders on Fridays. In this research, long measurement lead times, typical of previous AMS operations, completely idle the research until results are obtained.

I have designed rapid, parallel-sample processing that is done in conjunction with, and close to, the production of these samples in the biological laboratory. Rapid turnaround for projects like the immunoassay, and high throughput for whole-body tracing have been achieved. The preparation protocol has built-in safeguards, so that even minor cross-contamination of samples is eliminated by design rather than through conscious effort.

\section{GRAPHITIZATION}

Many analytical systems in biomedical research operate on vaporized samples, and the obvious mating of these technologies to an AMS source is still one of our goals. However, we continue to pursue graphite production for use in our cesium-sputter ion source, because the complete preparation of the graphite can be performed independently of the expensive accelerator time and far from the possible contamination of unrelated samples. If the combustion of the biomedical sample is accomplished directly at or in the ion source, gas-feed ion sources have the advantage of easy preparation prior to the time of measurement. Such arrangements are still impractical because of the dynamic range of our samples, the clearing time required to reduce cross-contamination in gas handling systems and the cleaning time required on individual gas targets (Bronk \& Hedges 1990). Measurement of a biomedical sample is complete in a total of 3-8 min (repeated $10 \mathrm{k}$ count measures, with standards every 5-10 samples), less time than is required to switch and clear some gas sources. Ion sources other than the cesium-sputter type may be required for full integration of the ion source and sample preparation.

Graphite produced by hydrogen reduction of $\mathrm{CO}_{2}$ over iron or cobalt provides the desired properties for rapid, accurate and precise AMS measurements (Vogel, Nelson \& Southon 1989), but the production includes unacceptable risks of cross-contamination from enriched samples (Vogel, Southon \& Nelson 1990). Hydrogen reduction requires the use of a common gas manifold and a limited number of reusable reaction vessels. The throughput could be increased by using detachable reaction vessels, but cross-contamination could remain in any reused reactors. Jull et al. (1986) and Slota et al. (1987) developed zinc reduction to provide precise, accurate ${ }^{14} \mathrm{C}$ dates. Several laboratories pursued gaphitization by zinc reduction of $\mathrm{CO}_{2}$ within a single, sealed tube (H. Polach, personal communication 1991). Slota and Taylor (1989) discuss one such method. Reduc- 
tion by zinc within a sealed tube is an excellent way to process numerous samples in parallel, but a few troubling properties need amelioration.

In some cases, the reduction over zinc fails to start or produces graphite very slowly. Graphite produced over zinc requires a longer time than its hydrogen-reduced counterpart to come to a stable ion current in our ion source. Bulk isotopic fractionations are larger for the zinc product than the hydrogen product. Finally, a carbonaceous gas phase involving the catalyst metal is present in zinc reduction, permitting the graphite to precipitate separately from the catalyst. In my own experience, filamentous graphite occasionally formed behind the zinc powder or on the glass container (rather than on the iron or cobalt catalyst), and a gas phase must be invoked to explain the deposition of graphite on a nickel foil described by Jull et al. (1986). Some of these effects could be explained by the production of iron pentacarbonyl $\left(\mathrm{bp} 103^{\circ} \mathrm{C}\right.$ ) or cobalt tricarbonyl, which result from heating finely divided metals in a $\mathrm{CO}$ atmosphere. As volatiles, they could interact directly with the zinc, where cobalt, at least, can form zinc-cobalt carbonyl (Lee, Burlitch \& Hoard 1967). It is possible that zinc, its oxide or other compounds are thereby incorporated into the graphite. Adding hydrogen to a sealed zinc reduction might reduce these problems, since the deleterious effects are seldom seen in hydrogen-reduction systems. However, hydrogen must be introduced with a low vapor pressure to achieve a proper seal on the evacuated reaction tube. Zinc can reduce water to produce the needed hydrogen, but water poisons the catalytic properties of the cobalt. I added the hydrogen as $\mathrm{TiH}_{2}$, from which I obtained satisfactory results.

\section{PARALLEL SAMPLE PREPARATION}

The isolated biomedical sample is usually transferred as a liquid or wet tissue to a new $6 \mathrm{~mm} \times$ $50 \mathrm{~mm}$ culture tube (borosilicate glass), that has been baked in air at $550^{\circ} \mathrm{C}$. We handle the tube with clean forceps to avoid contaminating the exterior. The sample is dried in a vacuum centrifuge or by flowing nitrogen. One adds wire-form cupric oxide to the dried sample, and places the tube inside a 9-mm (OD) quartz combustion tube that has a breakseal-point end. The tubes are evacuated, flame-sealed, and placed in an oven at $650^{\circ} \mathrm{C}$ for $2 \mathrm{~h}$. This low combustion temperature prevents the destruction of the quartz tube by diffusion from the glass culture tube. Boutton et al. (1983) showed that this temperature is satisfactory for measurements of isotopes in biological samples. (However, this does not fully combust some fats, sugars and other components that vaporize and condense on the walls of the tubes!)

Because many biomedical samples have unexpectedly high ${ }^{14} \mathrm{C}$ concentrations, I designed a disposable plastic manifold for the transfer of $\mathrm{CO}_{2}$ from the combustion tube to the reaction tube (Fig. 1). The manifold parts cost approximately US $\$ 1.50$ per sample, and one technician can assemble 100 or more manifolds in a day. The combustion tube is connected to the plastic manifold using 5/16" or 8-mm (ID) soft plastic tubing. The break-seal point must extend into the harder plastic of the "Y" for easy breaking. A glass-wool plug in the neck nearest the break-seal prevents small sherds of quartz from being blown over to the reaction vessel. A 9-mm (OD) Pyrex tube, prepared with the proper amounts of reactants (zinc, titanium hydride and cobalt powders) is connected to another leg of the "Y." Finally, the third leg is connected to a vacuum manifold, and the assembly is evacuated. The combustion tube is immersed in a dry-ice slush to condense water formed in combustion. After evacuation, a hose clamp blocks the plastic tubing between the "Y" and the vacuum, the point of the combustion tube is broken and the $\mathrm{CO}_{2}$ is cryogenically trapped in the reaction tube. The hose clamp is removed to evacuate any nitrogen before the reaction tube is sealed with a torch. The entire plastic manifold is then discarded. 


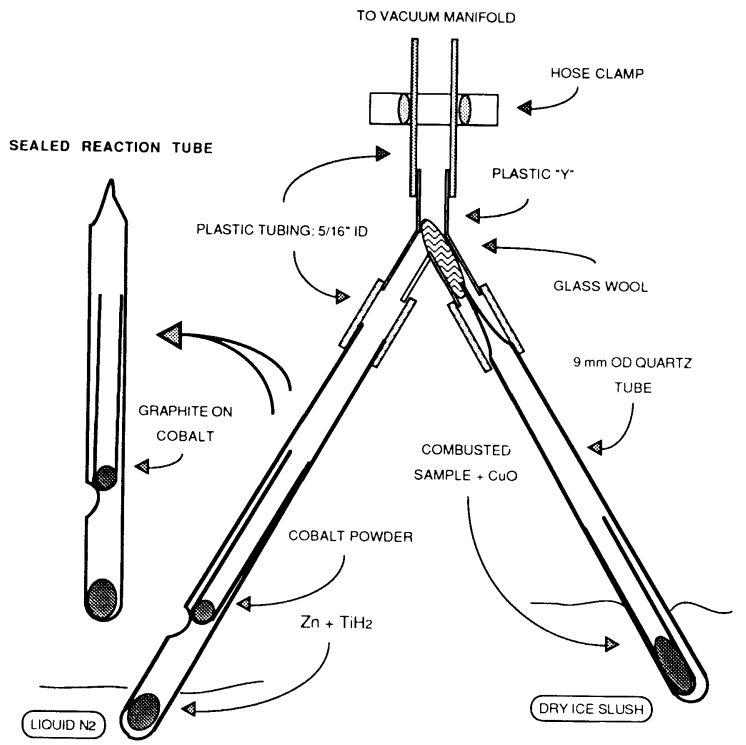

Fig. 1. A disposable plastic mini-manifold is used to transfer the $\mathrm{CO}_{2}$ from the combustion tube to the reaction tube. The quartz combustion tubes are made with a breakable point on one end. The borosilicate glass reaction tube has a dimple in one side to support the smaller tube containing the cobalt catalyst above the zinc and titanium hydride reductants.

The tubes are placed on their sides in a metal rack, and the rack is placed in a furnace at $500^{\circ} \mathrm{C}$ for $3 \mathrm{~h}$, and then $550^{\circ} \mathrm{C}$ for $2 \mathrm{~h}$. The number of samples processed is limited only by the size of the furnace. Different temperatures are not required on the zinc and cobalt. The product normally precipitates on or near the catalyst, forming filamentous graphite, which is easily packed into sample holders. Samples ranging in size from $150 \mu \mathrm{g}$ to $2 \mathrm{mg}$ have been successfully produced. An effect of internal gas pressure may occasionally cause samples with $>2 \mathrm{mg}$ of carbon in the 6-7 cc reaction tubes not to form graphite. A layer of "cobalt blue" material forms instead around the zinc and the titanium hydride. The graphite samples are stored in their reaction vessels until immediately prior to use. For each sample, the graphite is pounded into the central $1 \mathrm{~mm}$-diameter hole in the sample holder with a fresh drill stem.

\section{RESULTS}

Figure 2 shows the graphite yields as functions of the amounts of zinc and titanium hydride in the reaction tube. Forty $\mathrm{mg}$ or more of zinc $\mathrm{mg}^{-1}$ of carbon are required, as are 10-40 $\mathrm{mg}$ of the hydride. The data in the figure were obtained from samples reacting at $435^{\circ} \mathrm{C}$, and less hydride and zinc may be usable at higher temperatures. Production of methane in excess hydrogen may cause the reduced yields measured at high hydride levels. True yields may be higher than the maximum $80 \%$ in the figure. These yields are a comparison of the $\mathrm{CO}_{2}$ pressure in a poorly calibrated measure volume with the amounts of $\mathrm{CO}_{2}$ from recombustion before ${ }^{13} \mathrm{C}$-isotopic analysis. The ${ }^{13} \mathrm{C}$ fractionation is shown as a function of the yield in Figure 3. The seven samples of ANU sucrose that gave high yields were an average of $2.2 \%$ lighter than the initial sample. Even the larger isotopic shifts at lower yields would not markedly compromise the biomedical measurements. Some samples formed cobalt carbide instead of graphite, resulting in low ion outputs and 5-10\% errors in the ${ }^{14} \mathrm{C} /{ }^{13} \mathrm{C}$ ratios. Incomplete progression to graphite occurred about $10 \%$ of the time in the 300 samples that we initially made at a $435^{\circ} \mathrm{C}$ reaction temperature. Less than $5 \%$ of the samples fail to make graphite at the temperatures that we now use. Smaller amounts of cobalt can also force the precipitation of graphite. 


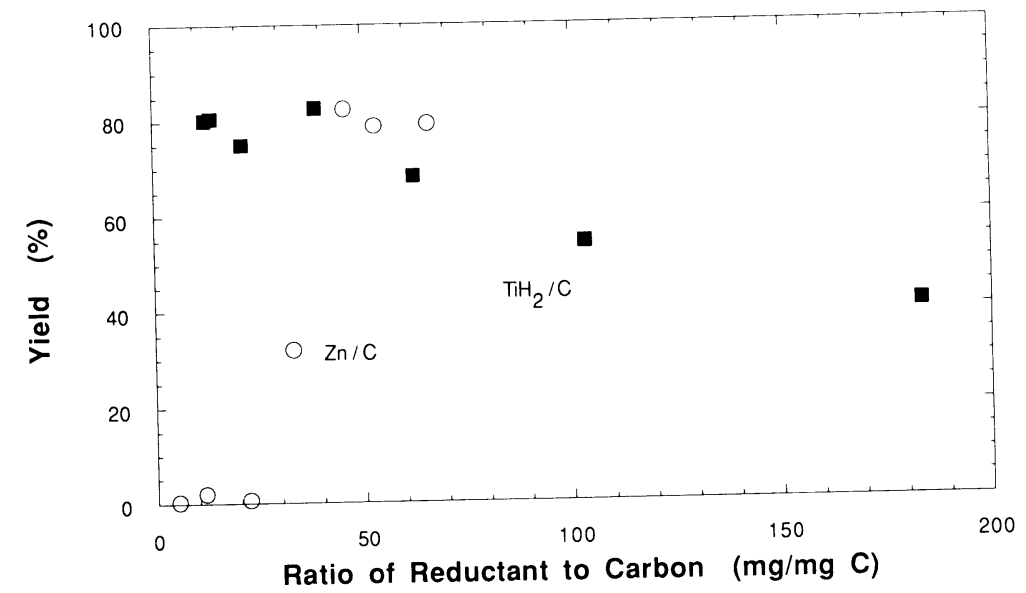

Fig. 2. The yield of graphite from $\mathrm{CO}_{2}$ in the sealed tube process is a function of both the zinc and the titanium hydride contents of the reaction tube. To obtain the variability with zinc, the $\mathrm{TiH}_{2}$ was held constant at $25 \mathrm{mg} \mathrm{mg}^{-1}$ carbon, whereas the zinc content was constant at $50 \mathrm{mg} \mathrm{mg}^{-1}$ carbon to determine the yield $v s$. the hydride. These data were taken at a reaction temperature of $435^{\circ} \mathrm{C}$, and the dependences may vary with temperature.

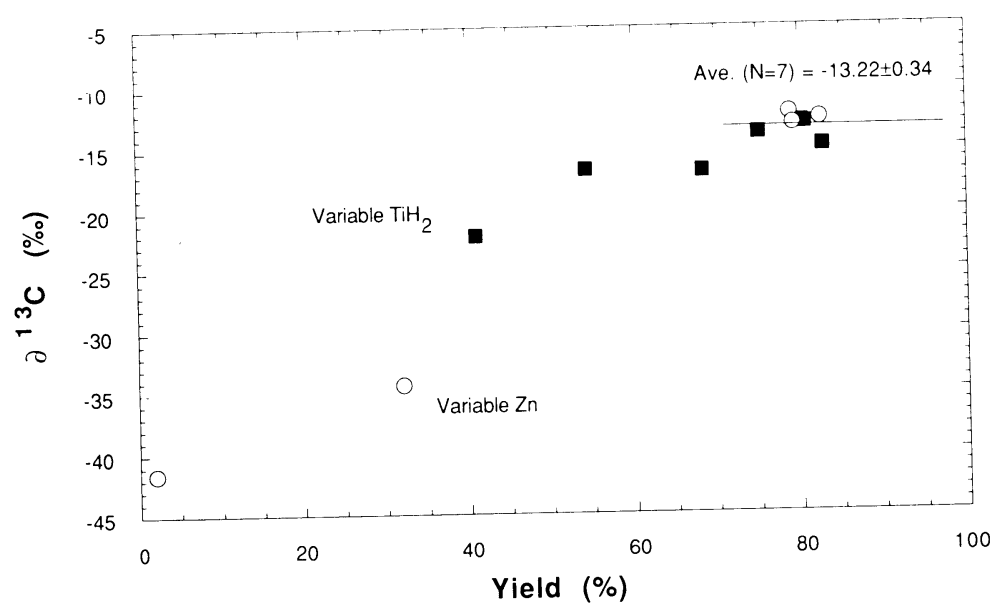

Fig. 3. The isotopic fractionation of the sealed tube process is a function of the graphite yield of the reaction. The seven highest yields showed a $2.2 \%$ o shift from the original material.

The graphites from this method produce ion beams in our source $\left(30-60 \mu \mathrm{A},{ }^{12} \mathrm{C}^{-}\right)$that are $50-90 \%$ those of graphites from hydrogen reduction. The ion intensity begins at a high level and there is little or no "burn-in" period. Figure 4 shows that the graphites are homogeneous by comparing successive measurements ( $10 \mathrm{k}$ counts each) and the final average of isotope ratios obtained from aliquots of five graphites produced by this method: three graphites were each split 50\%-50\% into separate sample holders and two graphites were each split 10\%-90\% in separate sample holders before measurement. The isotope ratio of the smaller or initial aliquot is divided by that of the larger or other aliquot to provide the data in the plot. Figure 4 also demonstrates the equivalence of this graphite to that produced by hydrogen reduction. I show the ratios of individual isotope measurements $\left(10 \mathrm{k}\right.$ counts each) and the final average ratio obtained from an aliquot of $\mathrm{CO}_{2}$ reduced by this method to the average obtained from two aliquots of the same $\mathrm{CO}_{2}$ reduced by hydrogen in our standard reactors. There were also no differences in isotope ratios between graphite samples of ANU sucrose that reacted for $5 \mathrm{~h}\left(\mathrm{D}^{14} \mathrm{C}=510 \pm 10 \%, \mathrm{~N}=5\right)$ and those that 


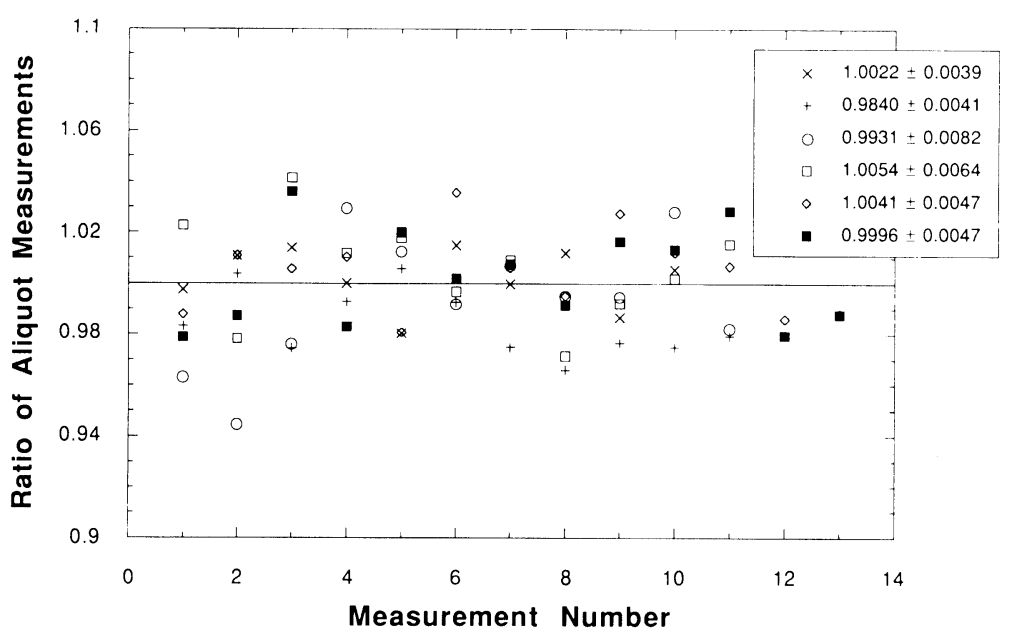

Fig. 4. Individual measurements of isotope ratios $\left({ }^{14} \mathrm{C} /{ }^{13} \mathrm{C}\right)$ were compared for different aliquots of the same materials. $\circ$, $\square, \diamond=$ The ratios of the isotope ratios between evenly divided aliquots of 3 separate graphites prepared in sealed tubes show homogeneity of graphite aliquots. $\times,+=$ The ratios of the isotope ratios between aliquots divided roughly as $10 \% / 90 \%$ show the dependence of the measurements on source presentation. $=$ The ratio of the isotope ratios between a sealed tube graphite and the average of two graphites prepared by the usual hydrogen reduction of the same $\mathrm{CO}_{2}$ show the equivalence of the two preparation processes. The width of the distribution indicates that we still have random fluctuations in our system greater than the $1 \%$ counting statistics would suggest, but the averages of all the measurements indicate that systematic errors are small, except in the case of one of the uneven splits.

reacted for $20 \mathrm{~h}\left(\mathrm{D}^{14} \mathrm{C}=516 \pm 12 \%\right.$ o, $\left.\mathrm{N}=4\right)$, when hydrogen reduced graphites were used as standards.

I do not expect this method to produce high-precision ${ }^{14} \mathrm{C}$ dates for other disciplines, because the processing background is $\sim 1 \mathrm{pMC}$ and variable. This background is not surprising, because the sealed reactors have a larger surface than our hydrogen reactors. We do not specifically clean the metal powders before use. In an attempt to clean and reduce all surfaces and the powders in situ before use, we baked prepared reaction tubes in a vacuum oven at $250^{\circ} \mathrm{C}$ in a 5 psia atmosphere of hydrogen overnight. The process modified the catalytic effects of the surfaces to such an extent that no graphite was produced.

\section{CONCLUSION}

This method of graphite preparation possesses all of the properties required to expand biomedical AMS measurements of ${ }^{14} \mathrm{C}$. We have kept a weekly cycle of 50-100 measurements and analyses, with all of the sample preparation being performed on 2 to 3 days a week. The plastic, disposable manifold has completely eliminated cross-contamination from the graphitization procedure. The ${ }^{14} \mathrm{C}$ concentrations in a recent series varied over many orders of magnitude $(100,000$ to $1.5 \mathrm{pMC})$ without contaminations. Whereas the procedure may never replace hydrogen reduction in terms of precision, other disciplines may also benefit from this technique, if they require large numbers of measurements at $1-2 \%$ precisions. 


\section{ACKNOWLEDGMENTS}

Work was performed under the auspices of the US DOE by Lawrence Livermore National Laboratory under contract W-7405-ENG-48. I would like to thank Erle Nelson and Bente Nielson for ${ }^{13} \mathrm{C}$ measurements and Sue Trumbore, Ken Turteltaub and John Southon for useful discussions. Kurt Haack nobly processes the deluge of biomedical samples into graphites.

\section{REFERENCES}

Bronk, C. R. and Hedges, R. E. M. 1990 A gaseous ion source for routine AMS radiocarbon dating. In Yiou, F. and Raisbeck, G., eds., Proceedings of the 5th International Conference on Accelerator Mass Spectrometry. Nuclear Instruments and Methods B52: 322-326.

Boutton, T. W., Wong, W. W., Hachey, D. L., Lee, L. S., Cabrera, M. P. and Klein, P. D. 1983 Comparison of quartz and Pyrex tubes for combustion of organic samples for stable carbon isotope analysis. Analytical Chemistry 55: 1832-1833.

Jull, A. J. T., Donahue, D. J., Hathaway, A. L., Linick, T. W. and Toolin, L. J. 1986 Production of graphite targets by deposition from $\mathrm{CO} / \mathrm{H}_{2}$ for precision accelerator ${ }^{14} \mathrm{C}$ measurements. In Stuiver, M. and $\mathrm{Kra}$, R. S., eds., Proceedings of the 12 th International ${ }^{14} \mathrm{C}$ Conference. Radiocarbon 28(2A): 191-197.

Lee, B., Burlitch, J. M. and Hoard, J. L. 1967 The crystal and molecular structure of $\mathrm{Zn}\left[\mathrm{Co}(\mathrm{CO})_{4}\right]_{2}$. Journal of the American Chemical Society 89(24): 6362-6363.

Slota, P. J., Jull, A. J. T., Linick, T. W. and Toolin, L. J. 1987 Preparation of small samples for ${ }^{14} \mathrm{C}$ accelerator targets by catalytic reduction of $\mathrm{CO}$. Radiocarbon 29(2): 303-306.

Slota, P. J. and Taylor, R. E. 1989 AMS ${ }^{14} \mathrm{C}$ analysis of samples from archaeological contexts: Pretreatment and target preparation. In Ericson, J. E. and Taylor, R. E., eds., University of California Accelerator Mass Spectrometry I, Proceedings of the First U.C. Conference on AMS. Institute of Geophysical and Planetary Physics, University of California, Lawrence Livermore National Laboratory. CONF-8602126: 30-43.

Turteltaub, K. W., Felton, J. S., Gledhill, B. L., Vogel, J. S., Southon, J. R., Caffee, M. W., Finkel, R. C., Nelson, D. E., Proctor, I. P. and Davis, J. C. 1990 Accelerator mass spectrometry in biomedical dosimetry: relationship between low-level exposure and covalent binding of heterocyclic amine-carcinogens to DNA. Proceedings of the National Academy of Sciences 87: 5288-5292.

Turteltaub, K. W., Knize, M. G., Healy, S. K., Tucker, J. D. and Felton, J. S. 1989 The metabolic disposition of 2-amino-1-methyl-6-phenylimidazo (4,5-b) pyridine (PhIP) in the mouse. Journal of Food and Chemical Toxicology 27: 667-673.

Vogel, J. S., Nelson, D. E. and Southon, J. R. 1989 Accuracy and precision in dating microgram carbon samples. Radiocarbon 31(2): 145-149.

Vogel, J. S., Southon, J. R. and Nelson, D. E. 1990 Memory effects in an AMS system: Catastrophe and recovery. Radiocarbon 32(1): 81-83. 